\title{
PROPUESTA CONCEPTUAL: LA PRODUCCIÓN SOCIAL DEL ESPACIO LOCAL ALREDEDOR DE MEGAPROYECTOS DE INFRAESTRUCTURA DE TRANSPORTE EN COLOMBIA
}

\author{
Julián de Jesús Pérez Ríos \\ Universidad de Antioquia, Medellín, Colombia \\ julianperezrios@gmail.com \\ ORCID iD: https://orcid.org/0000-0003-1422-0576
}

Recibido: 13/04/2017; Aceptado: 08/08/2019.

Cómo citar este artículo/Citation: Pérez Ríos, J. J. (2019). Propuesta conceptual: la producción social del espacio local alrededor de megaproyectos de infraestructura de transporte en Colombia. Estudios Geográficos, 80 (287), e022. https://doi.org/10.3989/ estgeogr.201939.019

RESUMEN: Por medio del análisis socioespacial se busca comprender el espacio producido por las grandes infraestructuras en su complejidad, generando procesos que involucran actores a diferentes escalas. Mientras los actores a escala internacional-global producen espacio promoviendo, asesorando y ejecutando megaproyectos de infraestructura (como la multinacional Odebrecht), y los actores a escala estatal-nacional subordinan el territorio, produciendo espacio concebido, los actores locales producen espacios vividos, generando acciones para la inclusión en la promesa de desarrollo implícita en los megaproyectos de infraestructura en transporte. El enfoque cualitativo sustentado en el trabajo de campo, las fuentes primarias y la investigación documental, permitió evidenciar las tensiones generadas por la intervención del espacio y cómo los habitantes defendieron su territorio por medio de acciones normativas y simbólicas, generando espacios de esperanza.

PALABRAS CLAVE: procesos globales; análisis escalar; movimientos sociales; megaproyectos; producción social del espacio; estudios socioespaciales.

\section{CONCEPTUAL PROPOSAL: THE SOCIAL PRODUCTION OF LOCAL SPACE AROUND MEGAPROJECTS OF TRANSPORT INFRASTRUCTURE IN COLOMBIA}

ABSTRACT: Through the socio-spatial analysis we sought to understand the space produced by large infrastructures in their complexity, generating processes that involve actors at different scales. While actors at the international-global scale produce space by promoting, advising and executing infrastructure megaprojects (such as the multinational Odebrecht), and actors at national-state level subordinate the territory, producing space conceived, local actors produce living spaces, generating actions for inclusion in the promise of implicit development in megaprojects of transport infrastructure. The qualitative approach based on the field work, the primary sources and the documentary research, allowed to show the tensions generated by the intervention of the space and how the inhabitants defended their territory by means of normative and symbolic actions, generating spaces of hope.

KEY WORDS: global processes; scale analysis; social movements; megaprojects; social production of space; socio-spatial studies. 


\section{INTRODUCCIÓN}

Al inicio de su primer período de gobierno, en el año 2010, los medios de comunicación registraban cómo el presidente de la República de Colombia Juan Manuel Santos anunciaba una estrategia de crecimiento para el país basada en el impulso de 5 «locomotoras» para el desarrollo: infraestructura, agricultura, vivienda, minería, e innovación. De entre estas locomotoras, el campo de la infraestructura tiene un lugar protagónico, en la medida que hace posible la movilidad y conectividad necesarias para el flujo de personas y mercancías, mejorando las condiciones para el crecimiento económico y gestando oportunidades para la generación de empleo.

De acuerdo con la necesidad de mejorar la malla vial del país, una de las más precarias de América Latina, se ha emprendido la implementación de las concesiones viales de tercera y cuarta generación, mediante la construcción proyectos como la «Ruta del Sol» y la adjudicación de los proyectos que conforman las «Autopistas para la Prosperidad». Así, el discurso del gobierno nacional se sintetizó en el lema «Prosperidad para todos», de manera que el crecimiento económico generado por las nuevas infraestructuras, la "mano invisible» y las demás locomotoras, asignaría a cada ciudadano su parte, su gota de desarrollo económico. Esta planeación y ejecución de proyectos estratégicos es una expresión a gran escala del espacio concebido, el cual hace parte de una triada conceptual (espacio percibido, concebido y vivido) que constituye una alternativa para comprender cómo se produce socialmente el espacio y las consecuencias de esa producción, de acuerdo con los planteamientos de Henri Lefebvre (1974).

La producción del espacio local durante la construcción de los megaproyectos ${ }^{1}$ de infraestructura es un fenómeno complejo, que implica la intervención de actores que configuran escalas de acuerdo a su capacidad de establecer relaciones de poder en términos de afectación, incidencia y subordinación. Actores a escalas como la internacional-global, la estatal-nacional y la local, producen el espacio en complejas interacciones, significando, defendiendo, apropiando y transformando el territorio. Teniendo en cuenta que todo conocimiento es parcial y situado (Haraway, 1995, p. 329), se busca aportar elementos de análisis para comprender la producción del espacio local en el marco de los megaproyectos de infraestructura en transporte.

A nivel analítico, la perspectiva economicista asume la sostenibilidad como base del desarrollo económi- co, aun cuando la destrucción de la naturaleza ha sido consecuencia del mismo. No obstante, el concepto de desarrollo sostenible ha sido plataforma discursiva de organismos nacionales e internacionales como la CEPAL, que a los tres pilares de sostenibilidad (ambiental, económico y social) ha añadido la dimensión institucional (Jaimurzina y Sánchez, 2017, p. 14) para integrar al Estado y los actores privados en la planeación del desarrollo.

Además, en cuanto a la relación de la sociedad y el Estado alrededor de las infraestructuras, se asume desde la CEPAL el concepto de governanza de la infraestructura: "la OCDE trabaja activamente en el tema de la gobernanza de la infraestructura, definiéndola como los procesos, herramientas y normas de interacción, toma de decisión y monitoreo, utilizado por las instituciones del Estado y sus contrapartes en el proceso de la provisión de los servicios de infraestructura a la sociedad" (Jaimurzina y Sánchez, 2017, p. 11). Teniendo en cuenta el gran impacto ambiental de los megaproyectos de infraestructura, es notoria la ausencia del tema de la protección ambiental en el discurso de la gobernanza y su dilución en el del desarrollo sostenible.

Si bien este es un trabajo de enfoque cualitativo, es importante tener en cuenta la experiencia de organismos como la Cepal en el tema de recursos naturales e infraestructura, mientras que para una visión cuantitativa de los proyectos de infraestructura en Colombia, es importante el trabajo de la Gobernación de Antioquia, Universidad de Antioquia, Universidad Pontificia Bolivariana con el apoyo del Sistema General de Regalías (2015).

La importancia de las concesiones de tercera y cuarta generación, (concesiones viales a privados por medio de los últimos modelos de licitación y contratación), es comprensible ante el atraso de la malla vial colombiana. Sobre las Autopistas para la Prosperidad, durante la presentación de los avances en la adjudicación de estas 9 concesiones viales de cuarta generación, en el año 2014 el Presidente Santos equiparaba estas obras con las que se construyen "en el primer mundo", tanto por sus características técnicas como por el presupuesto involucrado, tasado para el año 2013 en 26,2 billones de pesos, la cifra más alta aprobada por el Consejo Superior de Política Fiscal (Confis), involucrando vigencias futuras. Los beneficios de las Autopistas se traducirían en una disminución de los tiempos de viaje, en la generación empleo para unas 65.000 personas y en la reducción de los costos de transporte (República de Colombia, Presidencia de la República, 2014, p. 44). 
Por su parte, la Ruta del Sol es otro de los proyectos viales clave para Colombia, el cual busca mejorar la conexión entre el centro del país y la costa atlántica. Este proyecto consiste en el mejoramiento a doble calzada de una vía desde el municipio de Villeta en Cundinamarca (cercano a la capital Bogotá) hasta Ciénaga en Magdalena, atravesando ocho departamentos y 39 municipios del país con una longitud total de $1.071 \mathrm{~km}$, buscando incrementar la competitividad, promover el crecimiento económico y reducir el tiempo de recorrido (República de Colombia, Departamento Nacional de Planeación, 2009, p. 4).

La relevancia de una vía rápida para una población es proporcional a la distancia que separa la autopista de los centros poblados o cabeceras, y a la densidad demográfica de las zonas intervenidas. Por ello, los corregimientos (pequeños centros poblados con amplias zonas rurales, divisiones administrativas menores a los municipios) de La Floresta y Puerto Nus en el municipio de Maceo, y La Campana en el municipio de Aguachica (y quizás la misma cabecera municipal de Maceo en un futuro), viven un intensa relación de dependencia con las vías, ya que crecieron alrededor de la carretera número 45 o Troncal Oriente en el caso del municipio de Aguachica y la vía Medellín - Puerto Berrío en el caso de los mencionados corregimientos de Maceo.

A partir de la investigación realizada en el marco de la tesis de maestría en Estudios Socioespaciales del Instituto de Estudios Regionales de la Universidad de Antioquia, y con la participación en el proyecto Implicaciones de las Autopistas para la Prosperidad de la Gobernación de Antioquia, se realizó un análisis cualitativo que permite comprender la forma como los diferentes actores producen el espacio desde diferentes escalas, con una breve presentación de los espacios percibido y concebido, sobre el cual se mostrará la propuesta de subordinación territorial y espacio-tiempo de coyuntura, para luego destacar el espacio vivido como forma de respuesta de los actores locales ante la intervención de su territorio.

\section{Metodología}

Para lograr el objetivo de evidenciar la producción del espacio, se interpreta la realidad a partir de un marco teórico y conceptual donde se destacan, como estructurantes del relato, los planteamientos de Henri Lefebvre (1974, p. 54), de manera que el espacio concebido por actores a escalas global y estatal es contestado por los actores locales habitantes del espacio percibido, generando espacio vividos, espacios de esperanza (Harvey, 2003, p. 40) que defienden el territorio y que buscan la inclusión en la promesa de desarrollo implícito en la construcción e implementación de las grandes infraestructuras. Así mismo, se ha acogido la propuesta de análisis escalar de Taylor y Flint (2002, p 24), quienes proponen pensar las relaciones internacionales teniendo en cuenta las escalas Global, Estado-nación y localidad, mientras que los conceptos de experiencia y expectativa de Reinhard Koselleck (1993, p. 388) permitieron comprender al pasado y al futuro como dimensiones temporales de la producción del espacio-tiempo. Estos referentes teóricos fueron básicos para interpretar los datos obtenidos en la investigación de campo realizada en los municipios de Maceo (Antioquia, Colombia) y Aguachica (Cesar, Colombia). No se trata de un estudio comparado, sino de estudios de casos que se complementan, de manera que Maceo representa la expectativa, puesto que las Autopistas para la Prosperidad eran apenas un proyecto, mientras que Aguachica representó la experiencia por el paso de la doble calzada Ruta del Sol sector 2, construida por Consol (Consorcio Constructor Ruta del Sol, empresa del conglomerado Odebrecht). Finalmente, cabe destacar la función heurística de los conceptos de espacio-tiempo de coyuntura, generado por la construcción de las nuevas infraestructuras, y subordinación territorial, expresión de la coyuntura que define las relaciones entre los actores internacionales-globales, el Estado-nación, y la localidad. Se trata de una propuesta conceptual que permite formular o reforzar metodológicamente los estudios sociales sobre megaproyectos de infraestructura.

Así, por medio de tal marco interpretativo, se analizó a la luz del enfoque cualitativo la información obtenida en la etapa de investigación, básicamente, a partir de entrevistas y fuentes documentales recuperadas en un trabajo de campo realizado durante el segundo semestre de 2014,con más de 35 entrevistas en Aguachica, unas estructuradas y otras que terminaron más en un relato, algunas en profundidad y otras conversaciones cortas; al igual que en Maceo, donde se realizaron 19 entrevistas estructuradas y algunas no estructuradas, dada la necesidad de orientar la conversación sobre algo no sucedido. A continuación, en la Figura 1 se presenta la ubicación de zona de estudio. 
FIGURA 1

MAPA QUE MUESTRA LA UBICACIÓN DE LOS MUNICIPIOS DE MACEO Y AGUACHICA CON RESPECTO A LOS MEGAPROYECTOS VIALES AUTOPISTAS PARA LA PROSPERIDAD Y RUTA DEL SOL

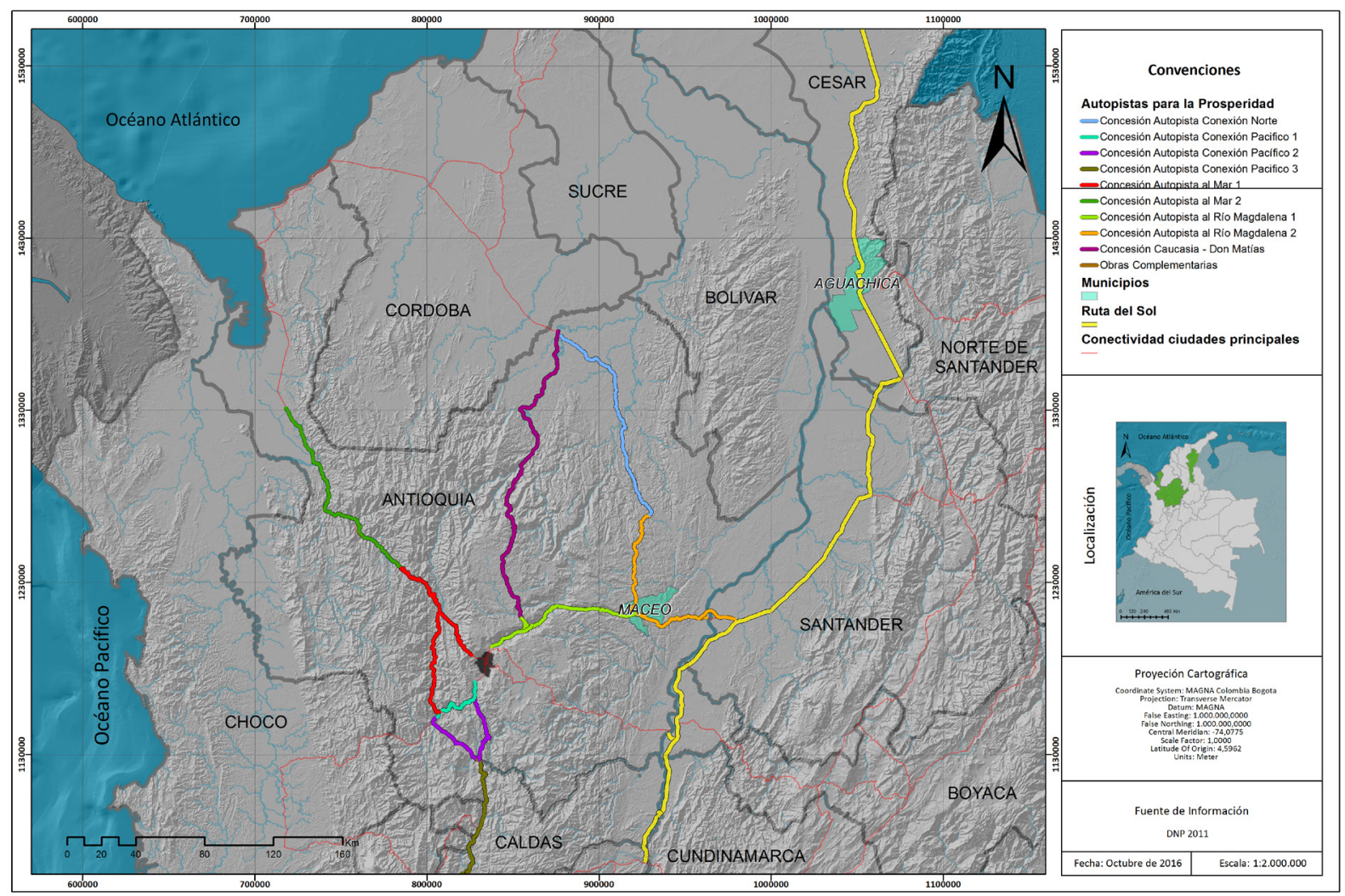

Diseño: Katlina Guarin

\section{EL ESPACIO CONCEBIDO}

Antes de señalar aspectos sobre la acción territorial de los actores a escala global y estatal-nacional, hay que anotar algunos elementos importantes al respecto. Partiendo de las ideas expresada por Taylor y Flint (2002, p. 24), quienes consideran que la ideología, a nivel del Estado, funciona como una cortina que separa la experiencia (local) de la realidad (global), es pertinente señalar que dicha ideología, en la interacción Global-estatal, está definida por las políticas neoliberales generadas a partir de lo que conocemos como el «Consenso de Washington» (Harvey, 2007, p. 27).

Como consecuencia de los procesos económicos neoliberales, los Tratados de Libre Comercio (TLC) han definido buena parte de la política económica internacional del Estado colombiano durante los últimos años, de manera que:

El peso del mercado exterior en la vida económica del país conduce finalmente una buena parte de los recursos colectivos para la creación de infraestruc- turas, servicios y formas de organización del trabajo frente a los recursos del comercio exterior, una actividad coordinada por el imperativo de la competitividad y localizada en los puntos más aptos para desarrollar estas funciones (Santos, 2008, p. 21).

Así, los TLC son la principal motivación para la construcción de los proyectos viales de tercera y cuarta generación, hecho registrado por los medios de comunicación e incluso aceptado por el Gobierno nacional, lo cual, unido a la asesoría de la IFC (Corporación Financiera Internacional) del Banco Mundial para el diseño del modelo de concesión, y al poder de las multinacionales de la construcción y de las grandes empresas Colombianas del ramo, ha implicado la convergencia de múltiples relaciones de poder sobre el espacio local.

En este proceso de intervención sobre el espacio, la integración regional dentro del país parece una consecuencia obvia. No obstante, la instalación de costosos peajes y la baja incidencia en las economías locales de las poblaciones intermedias, hacen 
que la relación costo beneficio en las escalas regional y local sea difícil de sopesar.

Finalmente, entre otras territorialidades, la escala estatal nacional gestiona el espacio mediante las licencias ambientales (ver Rodríguez, 2011), tema fundamental para comprender la forma como el Estado colombiano re-ordena el espacio durante la planeación e implementación de los grandes proyectos de infraestructura.

Las licencias ambientales comparten con el tema de adquisición de predios el lastre de ser consideradas como un obstáculo para el desarrollo, ante lo cual, el gobierno ha lanzado propuestas como un límite de 10 días para la adquisición de predios y un plazo de 30 días para proceder a la expropiación de acuerdo al avalúo catastral. Se trata en todo caso de medidas que buscan agilizar los procedimientos para favorecer los intereses tanto del gobierno nacional como de las concesionarias, en tanto el bien común prima sobre el particular y se tiene en cuenta la función social de la propiedad (República de Colombia, 2013, Documento Conpes 3760, p. 11).

\section{Espacio-tiempo de coyuntura}

Se entiende por espacio-tiempo de coyuntura a la transformación de las relaciones económicas y socioespaciales que ocurren en el momento y en el área de construcción e implementación inicial de los grandes proyectos de infraestructura, de manera que la población se ve enfrentada a procesos de afectación (costos y beneficios) y resiliencia. El espacio-tiempo de coyuntura estaría definido por las siguientes características:

- Implica una transformación física general del espacio que se acentúa proporcionalmente con la cercanía a las obras, en términos de paisaje, hábitat, ecosistema, territorio, recursos, usos del suelo, etc.

- Implica una fuerte alteración en el equilibrio relacional de los factores bióticos y abióticos, generando una percepción (objetivada) de crisis ambiental.

- Genera la producción o expansión de espacialidades urbanas asociadas al aumento de la circulación de dinero, a la demanda de servicios de alojamiento y al tiempo festivo, como "zonas rosa", zonas de tolerancia e infraestructura residencial y hotelera.

- Es un evento de corto plazo (menor a 3 años en la mayoría de los casos) en contraposición al largo plazo que implica la etapa de implementación (puesta en funcionamiento) de las infraestructuras.

- Está asociado a la subordinación territorial, de acuerdo a la cual, y según la normatividad, el Estado nacional tiene la potestad de definir la ubicación de los grandes proyectos de infraestructura sin tener en cuenta la participación de los municipios ni las poblaciones locales, generando una situación de sometimiento territorial tanto con relación al gobierno y las instituciones nacionales, como con relación a la empresa concesionaria que recibe facultades y atribuciones de ordenamiento territorial por parte del Estado.

- Se trata de un proceso de privatización del espacio, pasando del espacio público articulado a las dinámicas locales, al territorio privado corporativo (Silveira, 2007) que define nuevas relaciones sociespaciales con la población local.

- Se define económicamente a partir de una serie de implicaciones de gran incidencia en la economía local: por una parte, como una gran inversión de la empresa privada sobre una población determinada, en términos de generación de empleo, adquisición de predios y transformación física del espacio; por otra parte, como una gran afectación al patrimonio y al sustento económico de quienes ven alteradas sus relaciones históricas de dependencia y articulación con el espacio intervenido; además, como un aumento de la inseguridad por la atracción de la bonanza sobre delincuentes de otras localidades; y finalmente, como una incidencia sobre el costo de la vida en las localidades, produciendo fuertes aumentos en la propiedad raíz, los arriendos y la canasta familiar.

- Se trata de un espacio-tiempo comparable con el Kairós de los antiguos griegos, es decir, un contexto de oportunidad, de cambio, de alteración de los ritmos de la vida cotidiana. Es decir, el Kairós es una alegoría al cambio rápido (velocidad, pies alados); es un tiempo diferente, un espaciotiempo de fortuna que nos cambia la vida. Es el tiempo de Dios de la filosofía escolástica. Es, en suma, el espacio y el tiempo en el cual le cambia la vida a muchas personas, para bien o para mal.

Este conjunto de implicaciones locales en la etapa de construcción de grandes proyectos de infraestructura, en tanto grandes intervenciones sobre el espacio, se refleja en al caso de Aguachica, donde se 
muestran tanto los efectos como las respuestas locales frente a la construcción del proyecto vial Ruta del Sol 2. No obstante, hace falta presentar otros aspectos importantes para comprender el fenómeno.

\section{Subordinación territorial}

Para describir la relación de contención y jerarquía manifiesta tanto a partir de la normatividad como de su relación geográfica, se pueden nombrar las escalas como de primer orden (local), segundo orden (Estatalnacional) y tercer orden (global). Esta forma de nombrar al espacio facilita su incorporación en el discurso, ampliando el léxico disponible y enuncia en sí mismo una relación ordinal, donde lo local debe ser el primer objetivo de atención por parte de todas las escalas del poder y donde la relación de mayor a menor expresa la ya nombrada relación de contención y jerarquía.

Así, como fue evidenciado por Silveira (2007: 22), las escalas de segundo y tercer orden, como hegemónicas y como productoras de la realidad, ejercen subordinación sobre la escala de primer orden: «Predomina el orden global y el acontecer jerárquico, que subordinan las demás formas de acontecer, las otras formas de vida y de trabajo.» No obstante, es en primer orden en el cual se produce la interacción entre los diversos ordenes escalares para producir el espacio, en términos de diversas espacialidades, como espacios vividos, espacio-tiempo de coyuntura, hábitat, territorios, territorios corporativos, territorios subordinados, etc., los cuales describen un estado de las relaciones socioespaciales en un momento determinado.

Con esta introducción, se presenta la subordinación territorial como un concepto que busca explicar la relación de poder alrededor del espacio local entre el Estado central, la concesionaria (actor multilocal) y los actores locales (municipalidad y población) durante la etapa de construcción de las grandes infraestructuras.

La subordinación territorial define la potestad del Estado central sobre la ubicación y control de los grandes proyectos de infraestructura, marginando a las localidades de sus funciones de ordenamiento territorial y a la población de su derecho a la participación. Habría que decir, de entrada, que dicha subordinación no es de carácter constitucional, sino que se incorpora a la normatividad que la Constitución prevé para regular las competencias de las diferentes entidades territoriales. Así, sólo se encuentran en la Carta Magna con relación a las competencias territoriales, por un lado, el artículo 150 que le confiere al Congreso la fa- cultad de definir la división del territorio y establecer las competencias de las entidades territoriales; por otro lado, en el artículo 151 ordena también al Congreso el expedir las leyes orgánicas, entre ellas, "las relativas a la asignación de competencias normativas a las entidades territoriales», es decir, la Ley orgánica de ordenamiento territorial (LOOT). Con esto, se comprende que la Constitución delega el tema a la LOOT (Ley 1454 de 2011) y a la Ley 388 de 1997.

No obstante, en el artículo 287 de la actual Constitución colombiana se menciona un cuerpo básico de derechos de los municipios, los cuales se ejercerán de acuerdo "a los límites de la constitución y la ley»: 1. Gobernarse por autoridades propias; 2 . ejercer las competencias que les correspondan; 3. administrar los recursos y establecer los tributos necesarios para el cumplimiento de sus funciones; y 4. participar en las rentas nacionales. Esta remisión a otras normas que dicten las competencias "que le correspondan» muestra también cómo el ordenamiento territorial tiene dentro de la Constitución sólo los elementos más básicos (de acuerdo a su espíritu de marco general), delegando al legislador la competencia para expedir la normatividad correspondiente, como aparece en el artículo 288, el cual señala un aspecto muy importante del ordenamiento territorial colombiano: los principios que rigen la relación entre los tres niveles territoriales básicos:

ARTICULO 288. La ley orgánica de ordenamiento territorial establecerá la distribución de competencias entre la Nación y las entidades territoriales.

Las competencias atribuidas a los distintos niveles territoriales serán ejercidas conforme a los principios de coordinación, concurrencia y subsidiariedad en los términos que establezca la ley.

Estos principios se ponen en juego cuando se subordina el territorio local, ya que no existe una estrategia clara de cooperación, asistencia o acompañamiento por parte del gobierno nacional para las localidades en el contexto de la construcción e implementación de los grandes proyectos de infraestructura, situación que define en gran parte el conjunto de relaciones socioespaciales entre el Estado, la empresa privada y la población local, y que influye también sobre las posibilidades de articulación de las localidades a iniciativas estatales estimuladas por las demandas globales.

Continuando con la normatividad que define las competencias territoriales, la ley 388 de 1997, en su artículo 7으, enuncia las competencias en materia de ordenamiento territorial, asignándole al Estado cen- 
tral («la Nación») la potestad de ordenamiento en «asuntos de interés nacional», como la «localización de grandes proyectos de infraestructura.» (República de Colombia, Ley 388 de 1997). Por su parte, la Ley Orgánica de Ordenamiento Territorial 1454 de 2011, en su artículo 29 señala la «Distribución de competencias en materia de ordenamiento del territorio", y en su artículo 2 asigna a «la Nación» «b) Localización de grandes proyectos de infraestructura.», con lo cual tales proyectos someten tanto a municipios y departamentos a una situación de subordinación territorial frente al Estado central («la Nación») y frente a las grandes firmas constructoras, aun cuando este tipo de proyectos afectan de manera especial, espacial y socialmente, el nivel territorial de las localidades.

Puede afirmarse entonces que la forma en la cual la legislación ha asignado competencias territoriales al Estado central a expensas del poder de ordenamiento territorial del Estado local, excluye la participación del nivel municipal en las decisiones sobre megaproyectos de infraestructura, y termina omitiendo los principios constitucionales y legales que sustentan las relaciones entre las diferentes instancias territoriales: coordinación, concurrencia, subsidiaridad (estos los constitucionales), complementariedad, eficiencia, equilibrio entre competencias y recursos, gradualidad y responsabilidad (Artículo 27, Ley 1454 de 2011). ${ }^{2}$

La subordinación territorial se expresa también a nivel de los derechos individuales (congelamiento de bienes) y en temas como las licencias ambientales expedidas por la Autoridad Nacional de Licencias Ambientales ANLA desde el centro del país, llegando a conocer de manera muy superficial las condiciones y necesidades de las regiones y localidades.

No obstante, para comprender el espacio local es necesario un acercamiento a lo que identificamos como el espacio percibido, una breve caracterización de las realidades socioespaciales de los municipio de Maceo y Aguachica que permite perfilar los efectos y respuestas locales frente a los megaproyectos de infraestructura en transporte.

\section{EL ESPACIO PERCIBIDO}

Buscando una síntesis necesaria sobre el espacio percibido, en la Tabla 1 se resumen algunos de los principales factores que inciden en las dinámicas locales de los espacios en estudio, espacios constituidos por las trayectorias de la vida cotidiana, la espacialidad histórica e inmediata que nos hace parte de un lugar. ${ }^{3}$

TABLA 1

ALGUNOS ASPECTOS DEL ESPACIO PERCIBIDO, MUNICIPIOS DE MACEO (ANTIOQUIA) Y AGUACHICA (CESAR)

\begin{tabular}{|l|l|l|}
\hline Características & \multicolumn{1}{|c|}{ Maceo } & \multicolumn{1}{|c|}{ Aguachica } \\
\hline Extensión & $\begin{array}{l}\text { 431 kilómetros cuadrados (Gobernación de } \\
\text { Antioquia, Departamento Administrativo de } \\
\text { Planeación, 2016) }\end{array}$ & $\begin{array}{l}876.26 \text { kilómetros cuadrados (Alcaldía muni- } \\
\text { cipal de Aguachica, 2018). }\end{array}$ \\
\hline Poblamiento prehispánico & $\begin{array}{l}\text { Dataciones > 7.000 años de ocupación huma- } \\
\text { na (Pino Salazar, J. I. y Forero, J. C. (2002)) }\end{array}$ & $\begin{array}{l}\text { Grupos humanos tempranos alrededor del } \\
\text { río Magdalena y afluentes. (Alcaldía Munici- } \\
\text { pal de Aguachica, 2001). }\end{array}$ \\
\hline Poblamiento reciente & $\begin{array}{l}\text { Principios del Siglo XX, frontera agrícola y ex- } \\
\text { plotación de maderas }\end{array}$ & $\begin{array}{l}\text { Lugar de paso, zona de conexión entre el río } \\
\text { Magdalena y la cordillera Oriental. }\end{array}$ \\
\hline Población (Censo 2005) & $\begin{array}{l}\text { 7.630 habitantes (Gobernación de Antioquia, } \\
\text { Departamento Administrativo de Planeación, } \\
2016)\end{array}$ & $\begin{array}{l}\text { 82.335 habitantes (Alcaldía municipal de } \\
\text { Aguachica, 2018). }\end{array}$ \\
\hline Economía & $\begin{array}{l}\text { Ganadería, agricultura (cacao, café, frutales). } \\
\text { Conexiones geográficas }\end{array}$ & $\begin{array}{l}\text { Cultivos de algodón y otros a mediados del } \\
\text { Siglo XX, ganadería, agricultura extensiva, } \\
\text { petróleo (en el momento de la investigación). }\end{array}$ \\
\hline Violencia & $\begin{array}{l}\text { zueños. Berrío (río Magdalena), Medellín, } \\
\text { queñoste y Magdalena Medio Antio- }\end{array}$ & $\begin{array}{l}\text { Magdalena Medio, Sur-Sur de Bolívar, antigua } \\
\text { provincia de Ocaña (Norte de Santander). }\end{array}$ \\
\hline Cultura & $\begin{array}{l}\text { Presencia histórica de grupos guerrilleros y y } \\
\text { paramilitares, bandas criminales. }\end{array}$ & $\begin{array}{l}\text { Presencia histórica de grupos guerrilleros y } \\
\text { paramilitares, bandas criminales. }\end{array}$ \\
\hline
\end{tabular}

Fuente: elaboración propia a partir de fuentes documentales y trabajo de campo. 


\section{EL ESPACIO VIVIDO: EXPECTATIVA Y EXPERIENCIA}

La expectativa, el caso del municipio de Maceo: espacio local, representaciones del futuro y relaciones espaciales

Para comenzar, la investigación permitió establecer que existió una percepción generalizada en el municipio de Maceo, en términos de falta de información sobre el proyecto Autopistas para la Prosperidad, expresada en frases como: «Pues algo así, que van a pasar por aquí unas carreteras muy importantes pero no tengo mucho conocimiento.» (C. Bastidas, comunicación personal, 13 de julio, 2014).

Incluso los más informados consideran que ha faltado información precisa para comprender las implicaciones locales del proyecto, a la vez que se destaca la poca participación de las personas en las socializaciones.

Sobre la falta de información, ésta refleja la subordinación territorial del municipio ante la escala nacional, donde el Estado planifica el territorio con una mínima participación de la escala local, cuya población, entre la falta de información y la falta de experiencia, queda marginada de los proyectos de escala nacional, sin demandar participación.

Sobre el tema de la subordinación territorial, éste fue relacionado por los entrevistados, además de la falta de información, ${ }^{4}$ con la falta de propuestas del municipio en su plan de desarrollo y en su esquema de ordenamiento territorial, lo cual se relaciona con la ausencia de debates en el concejo sobre el tema, de acuerdo al entonces presidente del Concejo municipal. Esta falta de articulación de la Administración con la Corporación para la gestión del espacio muestra al municipio como un actor sin la relevancia necesaria, entendiendo la subordinación como condición para implementar los grandes proyectos de infraestructura, aunque se reconoce, paradójicamente, la necesidad de tener en cuenta la voz de los municipios, según lo expresado por algunos líderes locales.

Por otro lado, sobre la posible incidencia de las autopistas en los usos del suelo, no se esperan grandes cambios en la medida que buena parte del territorio municipal es usado en actividades relacionadas con la ganadería vacuna. No obstante, se encontró repetidas veces la idea de que las Autopistas afectarían la zona rural estimulando la instalación de fincas de recreo debido a la existencia de la autopista misma y a la mayor cercanía con Medellín, fenómeno que podría desplazar a pequeños propietarios, de acuerdo diferentes líderes locales entrevistados. Al respecto, se considera que, con relación a los predios que serán afectados directamente, mientras más pequeño el predio, mayor será la afectación espacial, lo cual refleja la vulnerabilidad de los pequeños propietarios que podrían terminar en el casco urbano o en la ciudad como "desplazados" del desarrollo. Esta situación de vulnerabilidad implica también para los pequeños propietarios rurales, y en un escenario de adquisición de predios, una transformación de sus prácticas asociadas a la vida campesina y de su seguridad alimentaria, debido a un probable desplazamiento hacia la cabecera municipal.

Por otra parte, Maceo carece de la mano de obra cualificada para aprovechar la demanda generada por las autopistas, aportando recientemente a diversas obras públicas cerca de 150 hojas de vida, muchas de ellas sin título de bachillerato. Esta situación significa que necesariamente la empresa tendrá que recurrir a mano de obra foránea para cubrir sus necesidades de personal, lo cual generará la llegada de forasteros al lugar, cuestión reconocida como posible factor de perturbación.

También se encontró que, al menos en un entrevistado, se reconoce que se dará una transformación del paisaje, pasando de una imagen rural a una caracterizada por el aspecto de modernidad que una autopista le imprime al espacio, en un proceso donde los territorios locales se adaptan a las necesidades nacionales. La importancia de la ruralidad en Maceo implica entonces pensar al municipio como un todo que incluye lo urbano, las veredas y los corregimientos, evitando reducir las relaciones socioespaciales al ámbito de la cabecera municipal que en sí misma hace parte de la ruralidad.

En cuanto al ordenamiento urbano, y en relación con la expectativa de crecimiento poblacional, se afirma que no está definido un suelo de expansión urbana en el municipio, lo cual, sumado a la dificultad que la topografía del paisaje colinado impone a la construcción, genera imaginarios sobre la apertura de una nueva calle en la plaza principal y la continuidad del poblamiento sobre las calles que aún lo permiten.

Y en términos ambientales, la crisis en el suministro de agua potable que se vivía en el municipio podría constituirse entonces en un factor influyente en la percepción de la población frente a las afectaciones ambientales generadas por la construcción de las Autopistas, y en las posibilidades de suministrar el servicio público de agua a una población que tendería a crecer. De hecho, se considera que las Autopistas 
mejorarán aspectos económicos en el municipio, pero generando a la vez un deterioro ambiental. Ante esta expectativa, se espera que la concesionaria cumpla con sus obligaciones de desarrollo sostenible y con la normatividad que regula su actividad, lo cual puede ser interpretado como una percepción positiva y de confianza frente a las grandes empresas y las instituciones, y que explicaría que ninguno de los entrevistados haya tocado el tema de las veedurías o de otro tipo de participación y control ciudadano.

Finalmente, la llegada de la multinacional cementera Cémex ha anticipado los efectos sobre el costo de la vida y el precio de arriendos y propiedad raíz, e incluso, el riesgo de prácticas de corrupción, ya que la compra de terrenos para el proyecto minero implicó la destinación irregular de $\mathbf{2 0}$ millones de dólares, hecho registrado por la prensa nacional. Así mismo, mientras que hasta hace poco tiempo el precio de la propiedad raíz en Maceo era relativamente bajo, se ha encarecido como efecto de la llegada de la empresa. Esta valorización del espacio comporta tanto aspectos positivos como negativos, de manera que un pequeño propietario tendría que pagar un mayor impuesto predial con sus escasos recursos, mientras que un gran propietario recibiría beneficios aumentando el valor de su propiedad.

Otra de las implicaciones imaginadas por los pobladores tiene que ver con la posibilidad de que la existencia de una nueva vía genere procesos de concentración de la tierra alrededor de la infraestructura. Esta idea estaría fundada en el hecho de que quienes poseen mayores recursos económicos buscaran posicionarse espacialmente para aprovechar las ventajas que ofrecen las autopistas.

Por otra parte, además de las implicaciones sociales mencionadas, algunos de los entrevistados reconocieron que las Autopistas generarán también afectaciones ambientales como desecación de los cuerpos de agua, erosión, deforestación, emisión de gases, contaminación auditiva y afectación a la fauna silvestre. No obstante, existe también la percepción de que la concesionaria implementará las acciones correspondientes para la mitigación de las implicaciones negativas sobre el medio ambiente.

Para terminar con el caso de Maceo, si bien se espera una gran incidencia de las autopistas sobre la cabecera municipal, los corregimientos de La Floresta y Puerto Nus se encuentran en una situación especial, debido a su relación socioespacial histórica y actual con la vía Medellín-Puerto Berrío, con afectación a la supervivencias de muchas familias dependientes de su interacción con los usuarios de la vía, de acuerdo con la transformación social y espacial directa sobre sus centros poblados y sus actividades económicas.

\section{La experiencia: el caso de Aguachica}

Siguiendo la ruta concebida desde la planeación del trabajo de campo, la primera aproximación a relación entre la Ruta del Sol y la población local se realizó recurriendo a la versión oficial, la de los representantes de las instituciones y funcionarios del Estado local. Lo que se encontró fue una relación de gran satisfacción con la incidencia del concesionario "Consol" sobre la población y el territorio, de manera que, de acuerdo a lo expresado por la Secretaría de Gobierno:

"la gente está contenta, no se han sentido, decir que ha traído problemas o algo es mentira, porque igual al contrario generaron empleos que la gente no tenía, entonces la economía pues ha subido un poco, no puedo decir que nos haya caído regalada plata pero sí." (Secretaría de Gobierno de Aguachica, comunicación personal, 25 de agosto, 2014).

Esta visión favorable contrasta con los evidentes efectos que hacen parte del espacio-tiempo de coyuntura generado por la megaobra, de manera que la narrativa del espacio concebido se impone como versión oficial de la realidad, ante la evidente influencia de la concesionaria sobre la política y la administración local que provoca una visión positiva sobre las consecuencias de la obra, de modo que la relación entre el municipio y la empresa habría sido:

Excelentísima, y en obras sociales ha apoyado muchísimo, el alcalde ha pedido y nos ha dado, la concesionaria nos reparchó 3 calles, 3 calles principales eso fue lo que le pidió el alcalde a cambio de obras sociales, les pidió paviméntenme tales y tales calles, para qué, excelentísimo. ${ }^{5}$ En lo que hemos necesitado en problemas comunes o problemas de orden de público y de maquinaria o que hemos necesitado suministro de combustible para la policía o algo, al máximo, mejor dicho en ese sentido muchísimo con el municipio en ese sentido, podemos decir que no tenemos queja alguna. (Secretaría de Gobierno de Aguachica, comunicación personal, 25 de agosto, 2014).

Las consecuencias positivas se sobreponen a las negativas, reproduciendo el discurso del desarrollo que prevé el bienestar de las poblaciones mediante intervenciones que inciden de lo general a lo particular, confiando el mejoramiento de las condiciones de vida de la población al comportamiento macro de la economía, asumiendo como ciertas predicciones 
económicas que son expectativas que no siempre se reflejan en la realidad: "Definitivamente este tipo de inversiones pues le dan una mayor dinámica a la economía, incrementa el flujo de visitantes, estimula los negocios que digamos van en primera escala relacionados con turistas, negocios de comidas, negocios de hotelería, se estimula mucho" (E. Vega, comunicación personal, 29 de agosto de 2014).

Frente a esta versión oficial, existe una versión paralela que describe un panorama de cooptación de las instituciones y el gobierno local por parte de la concesionaria Consol, empresa de la firma Odebrecht, reconocida a nivel mundial como una gran constructora y por los escándalos que han develado prácticas institucionalizadas de corrupción a todos los niveles, facilitadas por un modelo de intervención de arriba hacia abajo, que asume las implicaciones locales como externalidades económicas y a la población como sujetos pasivos sobre los cuales se impone la transformación de las relaciones socioespaciales locales: "El abordaje de arriba para abajo tiende a crear sujetos pasivos. Ese modo autoritario está basado en redes que cooptan élites locales, no establecen políticas compensatorias para aquellos impactados por los proyectos y no tienen preocupación con modelos y culturas locales." (Lins, 2007: 188) Se entiende por qué el espacio-tiempo de coyuntura es visto por un sector crítico de la población como "un espejismo" (D. Claro, J. Ojeda, comunicación personal, 26 de agosto, 2014), es decir, como una realidad engañosa, imagen de progreso y desarrollo que oculta la aridez del espacio concebido expresado en las relaciones desiguales entre la concesionaria, el Estado y la población.

El proyecto Ruta del Sol 2, operado por la empresa concesionaria Consol, en tanto espacio concebido, ha significado la generación de unos 4.000 empleos y la capacitación de unas 10.000 personas en Aguachica, en lo que se conoció como módulo básico («desarrollo de competencias integrales para la vida») por parte del Servicio Nacional de Aprendizaje -Sena-, aliado natural de los grandes proyectos de infraestructura en términos de capacitación y certificación de personal en competencias básicas y en habilidades técnicas en construcción, labores de oficina, oficios varios, etc. (D. Claro, J. Ojeda, comunicación personal, 26 de agosto, 2014).

Con respecto al empleo, por un lado, en la zona se ha valorado este proceso en cuanto implica la formalización del trabajo, por medio del cual, por ejemplo, un conductor de transporte de personal ganaba $\$ 780.000$ (cerca de 242 dólares) más horas extras
(J. Solano, comunicación personal, 25 de agosto de 2014), y mediante el cual se obtenía afiliación a la seguridad social como gran plus de la formalidad.

Por otra parte, y cuestionando la anterior perspectiva, algunos consideran que el empleo generado por la Ruta del Sol es de mala calidad por ser un empleo temporal, por cambiar las expectativas de muchos campesinos empleados estimulando la migración campo ciudad, y por implicar para los trabajadores, a partir de cierto momento de las obras, largos viajes que requieren madrugar y cuyo tiempo y desgaste no es compensado en la relación costo-beneficio. Con este panorama, muchos de quienes encontraron empleo en la Ruta del Sol terminaron por ahorrar y comprarse una motocicleta para participar del mercado local del "mototaxismo", volviendo de un sector formal a una actividad económica propia de la economía informal.

\section{Pequeños poblados en la carretera}

Antes de las obras, en el sito Los Columpios había montallantas, 2 hoteles, restaurantes, residencias y viviendas. Debido a las afectaciones de la Ruta del Sol, los habitantes se movilizaron e impidieron el paso de la obra por el lugar ${ }^{6}$ ya que se sintieron engañados debido a una promesa incumplida de reubicación. Con la pérdida de las tradicionales relaciones económicas con los usuarios de la vía, muchos de los pobladores de Los Columpios debieron cambiar de actividad económica debido al aislamiento que generó la construcción de la doble calzada, dedicándose muchos de ellos, entonces, a la venta de combustible venezolano a sabiendas de que se trata de una actividad ilegal, lo cual muestra que obras como La Ruta del Sol generan normalización en el empleo de forma temporal, pero también informalidad entre la población afectada de manera directa.

Situaciones similares en términos de desestructuración de la relación de los habitantes con la vía y con el medio ambiente social y natural ocurrieron en otros centros poblados como Juncal, Morrison y Norean, de manera que las personas vieron alteradas sus formas tradicionales de ganar el sustento diario. Así, Norean se había constituido en un lugar que las personas de Aguachica frecuentaban los días festivos para disfrutar del bosque y las aguas claras de la quebrada del lugar, atributos que fueron destruidos por el paso de la maquinaria.

El drama personal vivido por los habitantes de Norean y de otras zonas rurales de Aguchica se refleja en el caso de un campesino que, coincidencialmente, 
era el padre de uno de los funcionarios de la administración municipal. Dicho funcionario relató cómo su padre tenía un terreno con un pequeño negocio que le producía algunas ganancias. Con el dinero de la venta forzosa compro una vivienda en el casco urbano de Aguachica, donde perdió la posibilidad de obtener un ingreso, ya que no hubo manera de rehacer el pequeño negocio.

\section{El caso del corregimiento La Campana y el movimiento social}

El corregimiento La Campana, también conocido como Cerro La Campana o Cerro de los Chivos debe su existencia a la presencia de la vía y al tránsito de transporte pesado por el lugar, por lo cual, la construcción de una variante llevaría a la quiebra a los negocios del lugar, los cuales consisten, de acuerdo con la Asociación de Propietarios en los siguientes servicios: servicio de hospedaje (hoteles); taller de mecánica (formal e informal); taller de soldadura; monta llantas; reporte de carga de vehículos; restaurante; cafetería; venta de minutos telefónicos; engrase de vehículos livianos y pesados (formal e informal); parqueadero; almacenes de venta de lubricantes; almacenes de venta de repuestos; almacenes de venta de lujos para vehículos; estaciones de servicio; billares; ventas de lotería y chance; servicio de calibración de frenos; lavado de vehículos; y fruterías. (Asociación "Cerro de los Chivos», S.f.).

Es así como, considerando la construcción de la doble calzada y la variante (vía por fuera del casco urbano) como una amenaza a la estabilidad socioespacial, ambiental y económica del lugar, la población se organizó creando la Asociación de Propietarios Cerro de los Chivos, la cual ha gestionado la movilización social y ha constituido una red discontinua pero en constante comunicación, de actores sociales que cuestionan al proyecto Ruta del Sol y a la Concesionaria Consol como competencia, en tanto tienen intereses económicos similares (prestación de servicios de carretera), o reconocen al megaproyecto como perturbador de las relaciones socioespaciales y ambientales y como poderoso agente de intervención del orden espacial local, frente al cual, el Estado debe actuar como garante de los derechos de la población.

La organización de La Campana se identifica como "Asociación de Propietarios", mientras que al conjunto de actores sociales que cuestionan la Ruta del Sol, diseminados en las diferentes poblaciones en el ámbito regional y alrededor de la vía, se identifican como conciencia colectiva. De esta manera, aunque la organización social en el corregimiento La Campana se constituyó alrededor de problemáticas marcadamente locales, el descontento de múltiples actores sociales en diferentes localidades ha generado una red de actores que producen espacio vivido al confrontar al espacio concebido por el Estado central y la concesionaria, cuestionando la arbitrariedad de la subordinación territorial. Así, la red de la conciencia colectiva involucra personas ubicadas en las poblaciones cesarenses de San Alberto, San Martín, Aguachica (y sus mencionados corregimientos), La Gloria, Pelaya, Pailitas, San Alberto y Curumaní, junto con algunos centros poblados ubicados sobre la carretera y pertenecientes a estos municipios, como Las Vegas, Burro, Floresta, La Mata (donde se presenta además una explotación petrolera), Besote, Minas y Líbano. Esta red territorial, unida al involucramiento y el respaldo de las autoridades a nivel departamental, ha significado un fortalecimiento de la acción colectiva en su objetivo de luchar por sus derechos frente a la presión social y espacial de la empresa constructora y el Estado central.

Se comprende entonces que la emergencia de una conciencia colectiva en el área de influencia directa de la Ruta del Sol es una de las principales respuestas sociales ante los efectos de un megaproyecto de infraestructura en transporte, evidenciando la generación de espacios vividos y territorialidades que contestan al espacio concebido y la territorialidad subordinante del Estado central y la concesionaria. A continuación, en la Tabla 2 se presentan las acciones que la movilización social realizó en defensa de sus derechos.

\section{CONCLUSIONES}

Para cerrar, se presentan a continuación una serie de conclusiones de tipo conceptual y metodológico que permitan comprender la interpretación realizada en este ejercicio. Este trabajo implicó integrar diferentes conceptos que estructuraron el análisis, como la escala, la producción del espacio, la experiencia como eslabón entre el presente, el pasado y el futuro, y la expectativa como representaciones del futuro que producen espacios imaginados a nivel local, y que describen la percepción del riesgo, de la esperanza, de la oportunidad y de la transformación.

En tono de autocrítica y enunciando elementos que podrían mejorar la discusión y propuesta, se podrían incluir elementos que estuvieron fuera de los límites de la investigación, como el tema de humanizar el desarrollo y la posibilidad de generar desarrollos otros, reconociendo, por ejemplo, a la naturaleza como su- 
TABLA 2

ACCIONES SIMBÓLICAS Y NORMATIVAS DE LA MOVILIZACIÓN SOCIAL FRENTE A LA CONSTRUCCIÓN DE LA RUTA DEL SOL EN AGUACHICA

\begin{tabular}{|l|l|l|}
\hline \multirow{4}{*}{ Acciones simbólicas } & Acción & Resultado \\
\cline { 2 - 3 } & $\begin{array}{l}\text { Implementación de un discurso de negación y } \\
\text { satanización del proyecto de infraestructura }\end{array}$ & $\begin{array}{l}\text { Información y transmisión del descontento } \\
\text { con las obras a las poblaciones locales }\end{array}$ \\
\cline { 2 - 3 } & Marchas y plantones & Visibilización de la problemática \\
\cline { 2 - 3 } & Ocupación del lugar de avance de las obras & $\begin{array}{l}\text { Presión para lograr negociación con la } \\
\text { empresa }\end{array}$ \\
\hline \multirow{2}{*}{$\begin{array}{l}\text { Acciones } \\
\text { normativas }\end{array}$} & Derecho de petición & $\begin{array}{l}\text { Consolidar la ciudadanía como forma de } \\
\text { respuesta ante el Estado y la empresa. }\end{array}$ \\
\cline { 2 - 3 } & Acción Popular & $\begin{array}{l}\text { Visibilizar las condiciones reales de la } \\
\text { empresa frente a las comunidades }\end{array}$ \\
\hline
\end{tabular}

Fuente: información de interlocutores clave, municipio de Aguachica.

jeto de derechos, como en el caso de los ríos Atrato y Cauca en Colombia. Al tiempo, es importante pensar en la necesidad de cambiar en los discursos hegemónicos de la sociedad la idea del desarrollo sostenible y de la naturaleza en tanto recurso. También sería fructífero profundizar el análisis desde la perspectiva de la Antropología del Estado y aplicar un análisis del discurso por medio de información de prensa sobre la relación estado-empresa-movilización social. Así mismo, una aproximación desde la teoría del actor-red podría visibilizar el papel de las infraestructuras como agentes del desarrollo.

En este trabajo se pone en evidencia cómo las autopistas se constituyen en articuladoras del territorio, el cual se encuentra en disputa cuando florece la movilización social, con diferentes niveles de afectación: mientras más cercanía con las vías, mayores efectos sobre la población; mientras mayor densidad poblacional, mayores efectos socioespaciales, mientras más desconocimiento de la realidad social por parte del Estado y la empresa, mayor el descontento de las personas relacionadas con la vía. La movilización social ha sido un fenómeno eminentemente geográfico, en tanto responde a la relación de las personas con el territorio constituido por la vía y sus usuarios, y porque las personas buscan proteger los espacios cotidianos y naturales en su relación material y simbólica con las vías.

Con relación a las 3 escalas, la escala local se presenta como escala privilegiada para conocer e interpretar los fenómenos sociales, de manea que cobra sentido como unidad, con límites político-administrativos, con relaciones de identidad y pertenencia por parte la población y con dinámicas económicas particulares, constituyendo un espacio social observable y con una serie de relaciones hacia adentro y hacia afuera que definen su configuración socio-histórica.

No obstante, la escala local está sometida a la subordinación en el marco de los megaproyecto de infraestructura. Características como contención, subordinación y desigualdad, implican siempre tensiones y conflictos, los cuales se develan al analizar las interacciones entre actores que comparten o enfrentan intereses con los de otras escalas.

Así mismo, el Estado-nación funciona como escala articuladora entre lo local y lo global (Taylor y Flint, 2002). Como productor del espacio concebido, el Estado-Nación legitima su accionar implementando un aparato técnico y normativo que, bajo el principio de la preeminencia del interés general sobre el particular, favorece la acción del concesionario por encima de los intereses de las localidades. Sin embargo, tal dispositivo técnico y normativo no está respaldado en una institucionalidad y un despliegue de personal que alcance a reconocer la complejidad de la realidad local y que haga cumplir las normas en beneficio de los pobladores locales; al tiempo, la concesionaria es un actor multi-escalar de grandes dimensiones, difícil de controlar y de adaptarse a las necesidades de la población, por lo cual la normatividad y las directrices técnicas no alcanzan a regular las relaciones entre la población y la concesionaria, facilitando procesos de corrupción. Mientras que la escala global impone las condiciones, la escala estatal las respalda y las hace aplicar en el contexto local.

Y en términos analíticos, para sintetizar la relación entre las dimensiones a partir de las cuales se analiza la producción del espacio, se presenta la Figura 2 que 
ilustra sobre las relaciones entre los componentes de la tríada espacial:

Se entiende la tríada espacial de Lefebvre (1974) integrada por el espacio percibido, concebido y vivido, como forma de acceder a la realidad de la producción social del espacio. Y reconociendo la interacción que existe entre las tres instancias de producción del espacio, se han identificado las relaciones más importantes entre los elementos que configuran, en este caso, al espacio local, buscando una síntesis necesariamente excluyente pero con valor explicativo. Así, de acuerdo la Figura 2, el espacio percibido, que constituye la realidad local, es resignificado por los actores sociales, implementando estrategias de defensa del territorio; así mismo, el espacio percibido sufre una reconfiguración de las relaciones socioespaciales que lo constituyen, a partir de la intervención del espacio que implican los megaproyectos. Esta intervención, propia del espacio concebido, implica la acción de actores globales y nacionales, y de la empresa a cargo del proyecto como actor multilocal, todos ellos con intereses y funciones que confluyen en la transformación del espacio. Esta acción sobre el espacio local implica una interacción con los actores locales que genera tensiones, rupturas (como en los temas ambientales) y acuerdos sobre la forma como se interviene el territorio. De esta manera, se comprende la dinámica de producción del espacio como una interacción de factores más que una acción paralela de elementos independientes.

FIGURA 2

RELACIONES ENTRE LA TRÍADA ESPACIAL DE LEFEBVRE (1974)

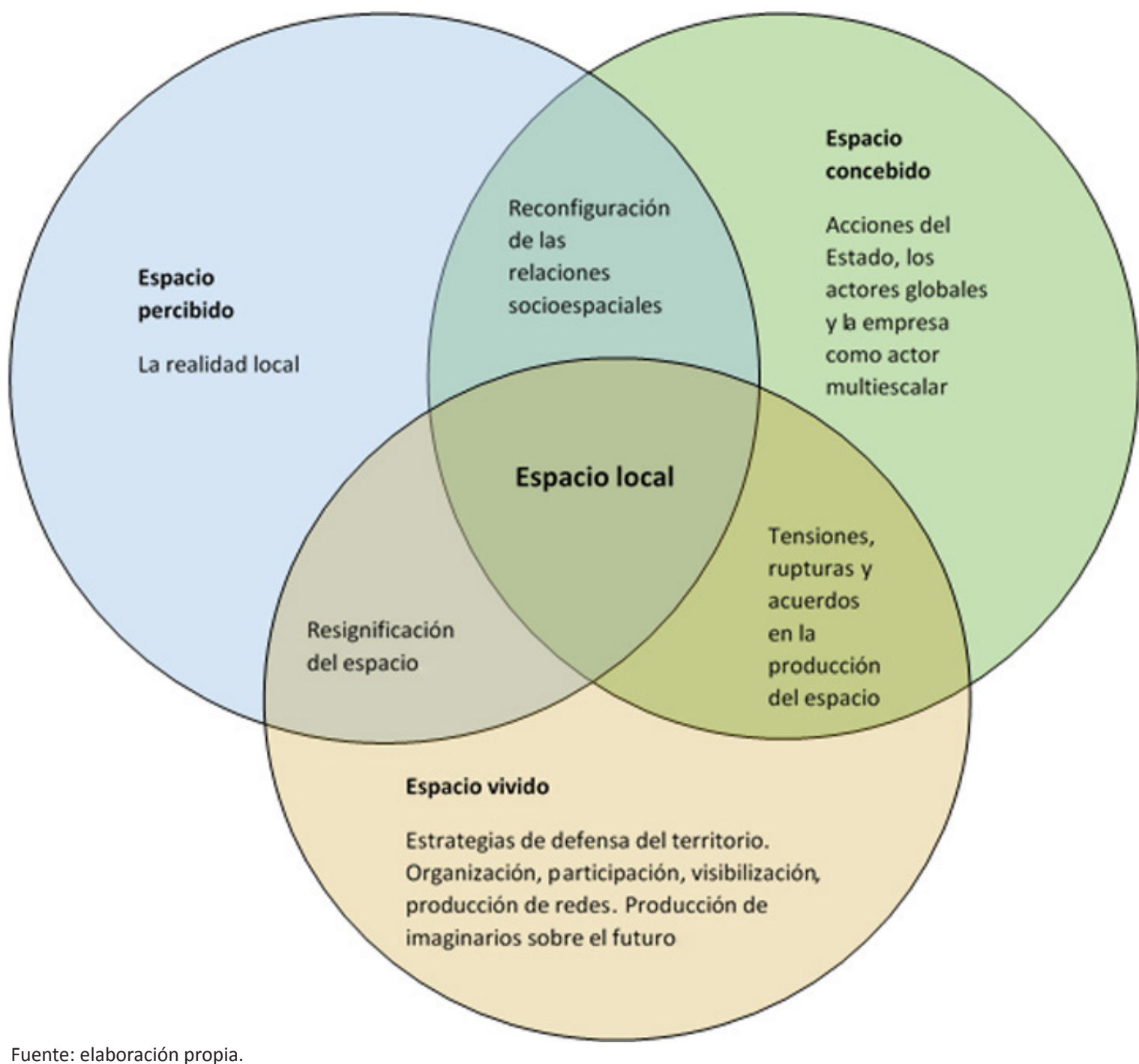


Por otra parte, abordando otros aspectos de las relaciones socioespaciales, se han propuesto los conceptos de espacio-tiempo de coyuntura y su componente de subordinación territorial, los cuales representan una forma de comprender las consecuencias de la construcción e implementación inicial de los megaproyectos de infraestructura. Su utilidad radica en que, como interpretación y caracterización de una porción de la realidad, permiten evidenciar y comprender las implicaciones de los megaproyectos sobre la población local, ofreciendo elementos para gestionar la articulación entre las diferentes instancias, niveles, actores y escalas implicados en estas grandes obras. El análisis de la coyuntura permite mostrar, así mismo, la necesidad de que el Estado y sus instancias a nivel nacional, regional y local brinden acompañamiento a la población local, y sobre todo a la afectada directamente, para que las grandes obras de intervención del espacio sean un factor de desarrollo local y no solamente de perturbación socioespacial.

Para cerrar, una propuesta metodológica para el estudio de los grandes proyectos de infraestructura, y como aporte desde los estudios socioespaciales, podría incluir las siguientes características:

- Se apoya en la producción del espacio y en categorías de análisis socioespacial.

- Ubica su metodología en el enfoque cualitativo, bajo la intención de conocer a fondo la realidad de las localidades, y que traduce el enfoque teórico en los estudios de caso de corte socioespacial.

- Hace uso de técnicas etnográficas, interpretación etnográfica y elementos de la teoría fundada para recopilar y sintetizar la información, como la generación de categorías de análisis. Las entrevistas, conversaciones, recorridos, visitas a luga-

\section{NOTAS}

1. El término "megaproyecto" se usa para designar a las grandes intervenciones del espacio que demandan, así mismo, grandes inversiones de capital y que tienen una gran incidencia en la vida económica y social de una región o país. De esta manera, autopistas, hidroeléctricas, minería a cielo abierto, grandes puertos, entre otros, se consideran como megaproyectos.

2. Para un ejemplo de cómo ha afectado la subordinación territorial a las entidades territoriales loca- res de documentación y el uso de los diarios de campo harán parte del acercamiento cualitativo a la realidad local.

- Tiene en cuenta las implicaciones de las escalas global y estatal-nacional en la producción del espacio local. A demás, incorpora el papel de actores de otras escalas, como la veredal, la departamental o la regional en la producción del espacio local, si es el caso.

- Tiene en cuenta otras experiencias similares que permitan comprender el fenómeno y hacer previsión de las posibles implicaciones negativas en otros casos.

- Implementa la expectativa como alternativa de análisis para trabajar en una dimensión temporal que involucre al futuro, así como la experiencia para comprender condiciones pasadas y presentes.

- Analiza las características propias del espaciotiempo de coyuntura y subordinación territorial generado por la construcción de un megaproyecto.

\section{AgRADECIMIENTOS}

Este artículo está basado en la tesis de maestría en estudios socioespaciales "Producción social del espacio local alrededor de dos megaproyectos de infraestructura de transporte. Los casos de Maceo (Antioquia) y Aguachica (Cesar)". Agradecemos el apoyo de la Estrategia de Sostenibilidad 2012-2014 del Comité para el Desarrollo de la Investigación (CODI) de la Universidad de Antioquia. Agradecemos también el apoyo del Grupo Estudios del Territorio del Instituto de Estudios Regionales (Universidad de Antioquia), y a Ana Carolina Guatame y Katlina Guarín por su apoyo técnico. Finalmente, agradecemos a los jurados por sus valiosos aportes para el cierre de este ejercicio.

les, se puede consultar la relatoría de la Sentencia C-149/10 de la Corte Constitucional de Colombia, la cual muestra por qué no se están cumpliendo dichos principios en la relación Estado-empresalocalidades. Dicha sentencia se promulgó frente a la situación de subordinación territorial en la que el Plan Nacional de Desarrollo (Ley 1151 de Julio 24 de 2007), y específicamente el artículo 79 ("Macroproyectos de Interés Social Nacional") puso a los municipios frente al poder ordenador del Estado central. 
3. Para ampliar la información de la Tabla 1, ver, para Maceo: Pino y Forero, 2002; Arcila, 1993; Cardona, 2014; Henao, 1997; Castaño, 2004; Sierra, 2013; Álvarez, 2012. Para Aguachica: Departamento del Cesar, 1992; Municipio de Aguachica, 2012; Alcaldía Municipal de Aguachica, 2001; Martínez e Iguarán, 2004.

4. Mientras lo demandado es una información oportuna y de calidad, las estrategias de socialización de la Agencia Nacional de Infraestructura -ANI- se orientan a hacer presentaciones generales, ignorando las particularidades de lo local y actuando cuando ya las concesiones han sido otorgadas (Ver: oficina de comunicaciones de la ANI, 26 de junio de 2014).

\section{REFERENCIAS BIBLIOGRÁFICAS}

Alcaldía Municipal de Aguachica (2001). Plan de Ordenamiento Territorial Aguachica, 2001-2010. Aguachica, Colombia.

Álvarez, F. (Alcalde) (2012). Plan de Desarrollo Municipio de Maceo 2012-2015. Reactivando el campo todos ganamos, Maceo, Colombia.

Arcila, M. T. (1993). Monografía de Maceo. Medellín.

Cardona, G. J. (2014). Historia del municipio de Maceo 1900-1950, Medellín, Colombia: L. Vieco.

Castaño, L. H. (2004). Reseña histórica de Maceo desde su fundación, Maceo, Colombia.

Departamento del Cesar (1992). América 500 años. Facetas histórico-folclóricas de la Noble Villa de San Roque de Aguachica. Aguachica, Colombia.

Gobernación de Antioquia, Departamento Administrativo de Planeación. (2016). Anuario Estadístico de Antioquia. Gobernación de Antioquia, Medellín. Recuperado de http://www.antioquiadatos. gov.co/index.php/anuario-estadistico-de-antioquia-2016

Gobernación de Antioquia, Universidad de Antioquia, Universidad Pontificia Bolivariana y Secretaría General de Regalías. (2015). Análisis de las implicaciones sociales y económicas de las Autopistas para la Prosperidad en el departamento de Antioquia. Medellín, Colombia: Gobernación de Antioquia.

Haraway, D. J. (1995). Ciencia, cyborgs y mujeres. La invención de la naturaleza, Madrid, España: Cátedra.
5. En entrevista con otro servidor público se estableció que fueron pavimentadas las calles 3 a entre 9 y 19 ; calle 5a entre 12 y 15 ; y calle $6 \underline{a}$ entre 10 y 20. Este trabajo, que involucra la actividad de pavimentación propia de la empresa y que por tanto no implica mayores costos, habría significado el mayor beneficio obtenido por el municipio de la empresa concesionaria en términos de responsabilidad social empresarial (J. Avendaño, comunicación personal, 29 de agosto de 2014).

6. Otras versiones atribuyen la parálisis de las obras en Los Columpios a la falta de la correspondiente licencia ambiental. No obstante, no se pudo verificar tal versión.

Harvey, D. (2003). Espacios de Esperanza, Madrid, España: Akal.

Harvey, D. (2007). Breve Historia del Neoliberalismo. Madrid, España: Akal.

Henao, L. D. (1997). Maceo: 100 años de historia, 1897-1997, Medellín, Colombia, Multicolor.

Jaimurzina, A. y Sánchez, R.J. (2017). Gobernanza de la infraestructura para el desarrollo sostenible en América Latina y el Caribe: una apuesta inicial. Boletín FAL, Cepal 354(2). Recuperado de https://repositorio.cepal.org/bitstream/handle/11362/41859/1/S1700455_es.pdf

Koselleck, R. (1993). Futuro pasado: para una semántica de los tiempos históricos, Barcelona, España: Paidós.

Lefebvre, H. (1974). La production de l'espace, Paris, Francia: Anthropos.

Lins, G. (2007). Poder, redes e ideología en el campo del desarrollo, Tabula Rasa, (6), enero-junio, pp. 173-193.

Martínez, S. e Iguarán, J. (2004). Orígenes. El Cesar y sus municipios, Bogotá, Colombia: Ápice.

Municipio de Aguachica Unidad Municipal de Asistencia Técnica Agropecuaria -Umata- (2012). Plan agropecuario y agroindustrial 2012-2015, Aguachica, Colombia: Umata.

Pino, J. I. y Forero, J. C. (2002): Ocupación humana y entorno natural en las cavernas del río Alicante, Maceo - Puerto Berrío, Antioquia. Medellín, Colombia: Corantioquia. 
República de Colombia, Departamento Nacional de Planeación. (2009). Documento Conpes 3571. Bogotá, Colombia: DNP.

República de Colombia. Presidencia de la República. (2014). Palabras del Presidente Juan Manuel Santos al término de la presentación de los avances del Megaproyecto 'Autopistas para la Prosperidad'. Santa fe de Antioquia, 22 de julio de 2014. Recuperado de http://wsp.presidencia.gov.co/Prensa/2014/Julio/ Paginas/20140722_09-palabras-Presidente-Santosal-termino-presentacion-avances-MegaproyectoAutopistas-para-la-Prosperidad.aspx

República de Colombia, Congreso de la República (1997). Ley 388 DE 1997, por la cual se modifica la Ley 9 de 1989, y la Ley 2 de 1991 y se dictan otras disposiciones, Bogotá, Colombia: Congreso de la República.

República de Colombia, Congreso de la República (2011). Ley 1454, 28 de junio de 2011, Ley orgánica de ordenamiento territorial. Bogotá, Colombia: Congreso de la República.

República de Colombia, Departamento Nacional de Planeación, Consejo Nacional de Política Econó- mica y Social (2013). Documento Conpes 3760. Proyectos viales bajo el esquema de asociaciones público privadas: Cuarta generación de concesiones viales, Bogotá, Colombia: Conpes.

Rodríguez, G. A. (2011). Las licencias ambientales y su proceso de reglamentación en Colombia. Bogotá: Foro Nacional Ambiental. Recuperado de http:// library.fes.de/pdf-files/bueros/kolumbien/08360. pdf

Santos, M. (2008). O Brasil. Térritorio e sociedade no inicio do século XXI, Río de Janeiro, Brasil: Record.

Sierra, M. (2013). Historia local de Maceo 1899-1953, Tesis de pregrado sin editar, Medellín, Colombia: Universidad de Antioquia.

Silveira, M.L. (2007): Los territorios corporativos de la globalización. Geograficando, 3(3), 13-26. Recuperado de http://www.memoria.fahce.unlp.edu. ar/art_revistas/pr.3665/pr.3665.pdf

Taylor, P. J. y Flint, C. (2002): Geografía Política. Economía-mundo, Estado-nación y localidad. Madrid, España: Trama. 